\title{
Is the hair nicotine level a more accurate biomarker of environmental tobacco smoke exposure than urine cotinine?
}

\author{
W K Al-Delaimy, J Crane, A Woodward
}

See end of article for authors' affiliations

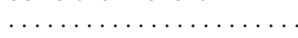

Correspondence to: Dr W K Al-Delaimy Department of Nutrition, Harvard School of Public Health, 665 Huntington

Avenue, Boston, MA 02115, USA: wael@hsph.harvard.edu

Accepted for publication 8 June 2001
J Epidemiol Community Health 2002;56:66-71

Study objective: The aim of this study was to compare the two biomarkers of exposure to environmental tobacco smoke (ETS); urine cotinine and hair nicotine, using questionnaires as the standard.

Design: A cross sectional study of children consecutively admitted to hospital for lower respiratory illnesses during the period of the study.

Settings: Three regional hospitals in the larger Wellington area, New Zealand.

Participants: Children aged 3-27 months and admitted to the above hospitals during August 1997 to October 1998. A total of 322 children provided 297 hair samples and 158 urine samples.

Main results: Hair nicotine levels were better able to discriminate the groups of children according to their household's smoking habits at home (no smokers, smoke only outside the home, smoke inside the house) than urine cotinine (Kruskall-Wallis; $\chi^{2}=142.14$, and $\chi^{2}=49.5$, respectively $(p<0.0001)$ ). Furthermore, hair nicotine levels were more strongly correlated with number of smokers in the house, and the number of cigarettes smoked by parents and other members of the child's households. Hair nicotine was better related to the questionnaire variables of smoking in a multivariate regression model $\left(r^{2}=0.55\right)$ than urine cotinine $\left(r^{2}=0.31\right)$.

Conclusions: In this group of young children, hair nicotine was a more precise biomarker of exposure to ETS than urine cotinine levels, using questionnaire reports as the reference. Both biomarkers indicate that smoking outside the house limits ETS exposure of children but does not eliminate it.
D eveloping appropriate measurement methods for environmental tobacco smoke exposure (ETS) (also known as second hand smoke) has attracted increasing interest as a result of the accumulating evidence of the ill effects of ETS on human health. Questionnaires have been the usual method used to assess exposure of ETS and its association to illness. However, questionnaires have drawbacks such as recall bias and low sensitivity, which may lead to inaccuracy in the interpretation of associations between ETS and illness. ${ }^{1}$

Biomarkers are becoming increasingly popular for ETS exposure measurement, as they avoid many sources of bias and may provide greater sensitivity than questionnaires. Urine cotinine levels have been suggested as the gold standard for ETS exposure measurement for these reasons. ${ }^{2}$ Undoubtedly, urine cotinine is currently one of the more widely used biomarker for ETS assessment, ${ }^{2-10}$ but it is affected by inter-individual variability in cotinine excretion levels for similar exposures and a relatively short half life of 20 hours. ${ }^{6711}$ Cotinine in saliva is subject to similar disadvantages in addition to the artificially high estimates of cotinine in saliva compared with serum levels because of the ability of the salivary glands to concentrate this metabolite. ${ }^{12}{ }^{13}$ Several studies have found significant misclassification when using salivary cotinine for ETS exposure assessment, ${ }^{14-16}$ and hence cotinine in urine has been generally favoured over cotinine in saliva. ${ }^{8}$

The more recently developed method of nicotine assay in hair, is less affected by the daily fluctuation of ETS exposure (because of the slow growth rate of hair), and it provides a long term exposure history because each $1 \mathrm{~cm}$ of hair represents approximately one month's exposure. ${ }^{17}$ However, chemicals applied to the hair (bleaching) and inter-individual differences in the rate of hair growth may affect nicotine levels. ${ }^{18}$

In this study we aimed to compare hair nicotine with urine cotinine by assessing the relation of these two "epidemiologi- cally cost effective" biomarkers with questionnaire assessment of ETS exposure assessment. Although questionnaires are not considered a gold standard, they have face validity and have been used for describing most of the associations between ETS and illnesses.

\section{METHODS}

The findings were part of a study looking at children admitted to hospital with respiratory illnesses and the effect of ETS on their illness. All children aged 3 to 27 months admitted to three hospitals in the Wellington region with a diagnosis of acute respiratory illnesses were involved in the cross sectional survey. The study was carried out over 14 months from August 1997 to October 1998 and involved 351 children. Twenty nine children did not provide hair nicotine or urine cotinine and were therefore excluded. Median age was 10 months, 65\% were male.

For parents agreeing to participate in the study, a 5-10 minute interview was carried out with them to record information about history of smoking in the house. The questionnaire was concerned with reported smoking habits of household members in the past six months. The average daily number of cigarettes smoked for each household member and visitor were categorised into five categories $(1-5,6-10,11-15$, $16-20,>20$ ) to represent the average ETS exposure dose for children. In an earlier study, it was noted that it is difficult for participants to specify an exact daily cigarette consumption, therefore participants were asked to choose between the above five categories, to assist them to identify daily smoking habits of household members and visitors.

The number of cigarettes smoked by parents was divided into those smoked at home and those smoked elsewhere. This was important, because the interest in this study was mainly in the exposure of children in the house. Furthermore, 
frequency of smoking inside the home was recorded (always, not always, occasionally, or never smoke inside the house) to compare the ability of hair nicotine and urine cotinine to discriminate ETS exposure in relation to smoke free and non-smoke free houses. Those who reported that they never smoked inside the house were considered the "outside" smokers and the rest were considered the "inside" smokers. Another possible place of exposure of children to ETS other than the home is the family car. A question was therefore included regarding exposure in the car and measures that the parents may have undertaken to avoid or decrease exposure. In addition, the primary caregiver was asked to estimate the exposure from other household members and visitors by reporting the number of cigarettes they smoked.

Other regular exposures of children outside the house were estimated by recording the number of hours the child regularly spent outside the home per week and the proportion of that time the child was thought to be exposed to ETS.

Afterwards, a hair sample was collected by cutting 10-50 $\mathrm{mg}$ of hair from the child's scalp. The hair sample was held in one bunch and put in a coded small paper envelope with the cut end of the hair in first. The ward nurse in charge of the patient was then requested to collect a urine sample from the child. The collected urine samples were immediately stored in the freezer at the laboratory or ward. Because of the difficulty in collection of urine samples from infants (the nurses too busy, or the sample contaminated with faeces), only 158 urine samples were collected. In contrast, 297 hair samples were collected from the participating children. A total of 133 children provided both hair and urine samples.

\section{Analysis of hair and urine samples}

Hair samples were analysed by the high performance liquid chromatography with electro chemical detection (HPLC-ECD) method, developed in our local laboratory. ${ }^{19}$ The proximal one centimetre of the hair sample was cut for analysis. Urine samples were analysed for cotinine by ELISA through the Community Paediatric Unit in Christchurch. The ELISA method has been used in previously published studies, ${ }^{20}{ }^{21}$ and its results well correlated with other methods..$^{22}$ All urine samples were adjusted for urinary creatinine. Both biomarkers incurred similar cost per sample. The lower detection limits for the HPLC and ELISA methods for nicotine and cotinine respectively were: $0.05 \mathrm{ng}$ nicotine $/ \mathrm{mg}$ of hair, and $0.02 \mathrm{ng}$ cotinine/mg creatinine.

The study protocol was approved by the Wellington Ethics Committee. In addition, a council of Maori (Kaumatua Kaunihera of Tangata Whenua of Wellington area) gave consent for the study to involve cutting hair from Maori subjects.

\section{Data analysis and power calculations}

Hair nicotine and urine cotinine levels were compared by first determining the strength of the relation between hair nicotine and questionnaire assessment, followed by determining the strength of the relation between urine cotinine and the same questionnaire variables. Differences between the two relations were then described. Hair nicotine and urine cotinine levels were also directly compared.

Thirteen children who provided urine samples had undetectable cotinine values. They were assumed to have $0.01 \mathrm{ng}$ cotinine per mg creatinine for the purpose of analysis. All main analyses for hair nicotine measure of exposure were re-run using the sample of children who had provided both urine cotinine and hair nicotine $(n=133)$ to assess the exclusion/ inclusion effect of children who provided hair samples but no urine samples. There were no appreciable differences in the results when excluding these children, and thus, for hair analyses results all 297 samples were used.

Non-parametric tests (Wilcoxon, Kruskall-Wallis) were used for assessing the difference in the nicotine and urine lev- els between children according to different exposure variables. Spearman rank correlation was used to describe the strength of correlation between the biomarkers and questionnaire reports of exposure. The coefficient of variation $\left(r^{2}\right)$ was determined from the output of the regression models to estimate the variability in hair nicotine and urine cotinine predicted by reported exposure variables.

For optimal prediction of the regression model, grouped forward stepwise selection of reported contributory variables to total ETS exposure was used in building the regression model for assessment of the relation of nicotine and cotinine to these variables. The number of cigarettes smoked by the mother, father, other household adults, and visitors were initially included. Then dichotomised presence or absence of exposure from the car and the place of smoking (outside the house, or inside) were later included. All non-significant variables $(p \geqslant 0.05)$ in the model were excluded. The same variables were used for both nicotine and cotinine regression models.

The study sample size (322) would have $80 \%$ power to detect a correlation of approximately 0.15 or higher, between hair nicotine and urine cotinine at the $\alpha=0.05$ confidence interval.

\section{RESULTS}

\section{Hair nicotine}

From the 322 children involved in the study, 297 provided hair samples. Nicotine in hair ranged from 0.19 to $47.82 \mathrm{ng}$ nicotine/mg hair.

Three main variables were used for questionnaire assessment of exposure to ETS: home exposure status (no reported exposure, smoking only outside the home, smoking inside the home), number of households and visitors who smoked, and the number of cigarettes smoked by parents, other household members and visitors.

Table 1 clearly shows a positive trend of nicotine hair levels among children according to whether they were reported to be living in houses where there were no smokers and no visitors smoked inside the house, in houses where smokers and visitors smoke only outside the house, or in houses where smokers smoke inside the house. There was a statistically significant difference in hair nicotine levels between those groups (Kruskal-Wallis; $\chi_{\mathrm{df}=2}^{2}=142.14 ; \mathrm{p}<0.0001$ ). Figure 1 displays six histograms according to the three reported exposure groups of children; the frequency of children in each group are distributed according to hair nicotine and urine cotinine levels categories. The group of children who are exposed at home are clustered at the higher levels of hair nicotine, while those not exposed are clustered on the other end of the scale, and the group with smokers who smoked outside home are intermediate. This was not the case for urine cotinine.

There was a large difference between the hair nicotine levels of children living with smoking mothers and those living with non-smoking mothers (Wilcoxon; $\chi^{2}=118.83$; $\mathrm{p}<0.0001)$. A similar but weaker pattern was observed in relation to father's smoking habits (Wilcoxon; $\chi^{2}=44.3$; $\mathrm{p}<0.0001$ ). The mean hair nicotine level of children who had smoking mothers but non-smoking fathers was higher than that of children whose fathers smoked while their mother did not (fig 2). Figure 2 also shows that children who had both parents smoking had higher nicotine levels than if only one smoked.

When the number of smokers in the household was compared with the nicotine levels in the hair of children, there was a correlation of $r=0.69(\mathrm{p}<0.0001)$. The correlation was slightly stronger $(r=0.72)$ when the number of visitors who smoked inside the house were added to the household smokers. Furthermore, the hair nicotine levels of children varied with the categories of the number of household smokers, 

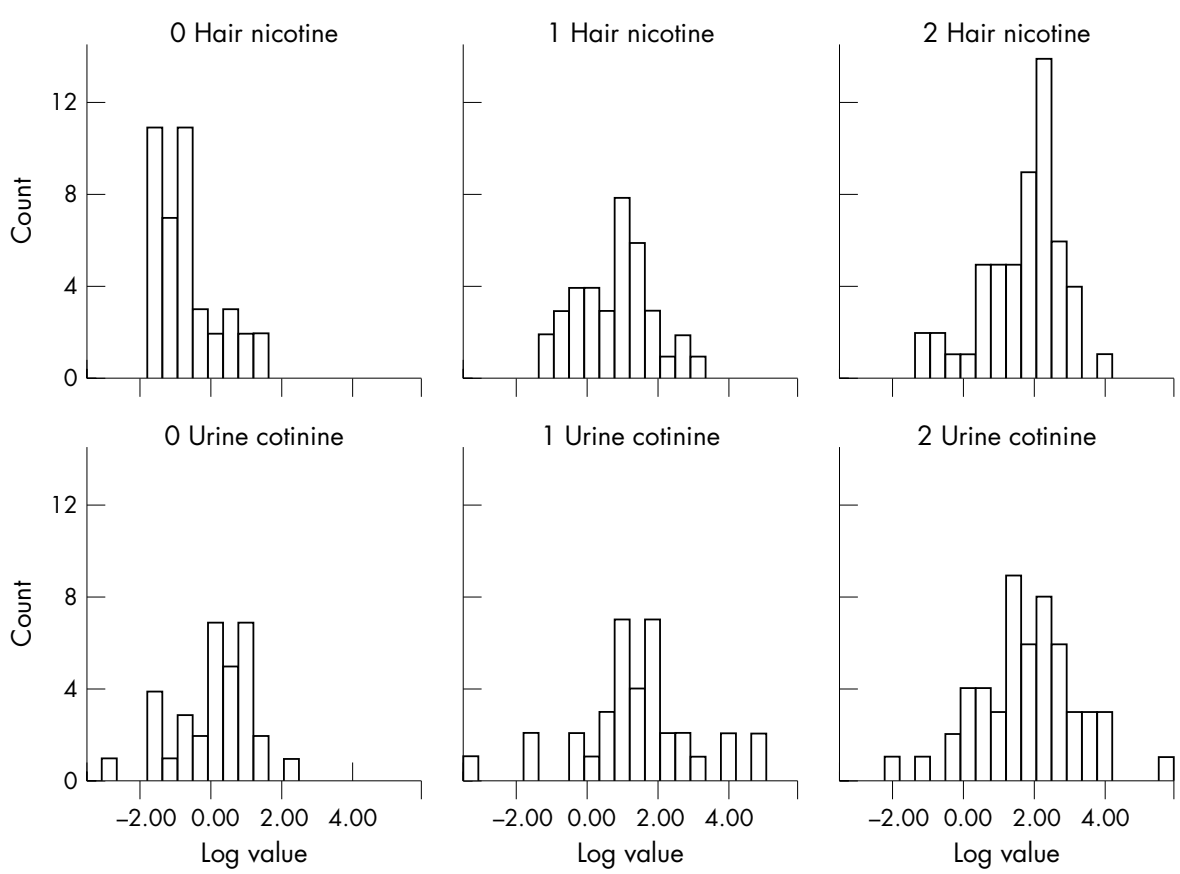

Figure 1 Histogram distribution of hair nicotine and urine cotinine levels by the reported history of smokers in the house among 133 children who provided both hair and urine samples. $Y$ axis is frequency of subjects in each category, $X$ axis is the categories of logarithm hair nicotine and urine cotinine levels, the six charts represent children according to their reported exposure group $11=$ children who live in homes with no smokers and no visitors smoke inside the house, 2 = children who live with smokers but no smoking takes place inside the house, $3=$ children who live with smokers who smoke inside the house).

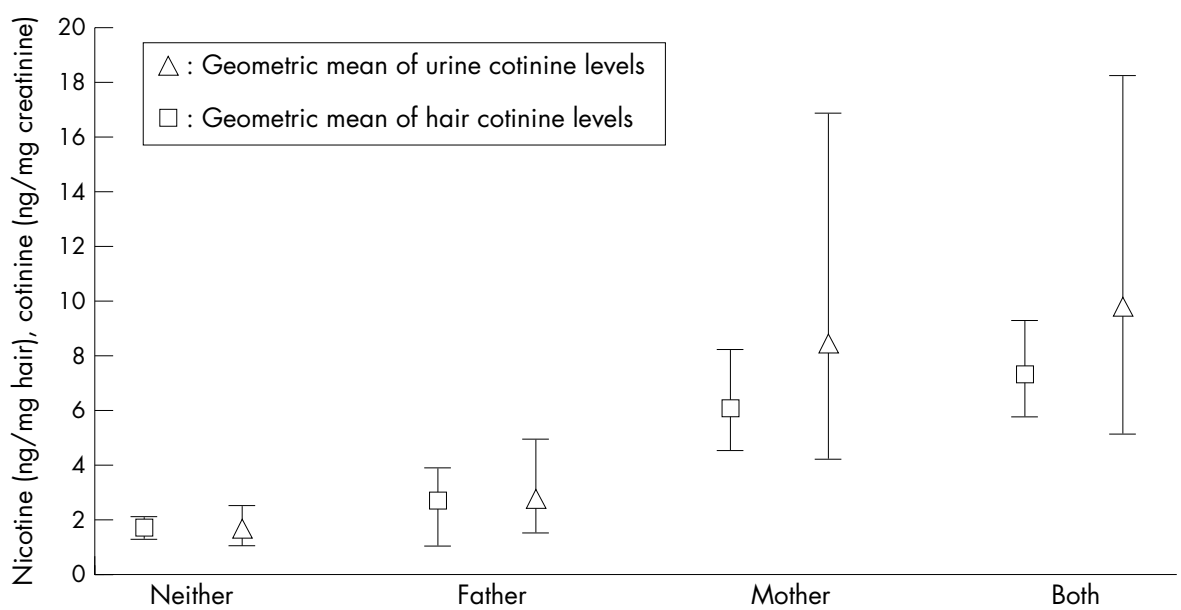

Figure 2 Geometric means (and 95\% confidence intervals) of hair nicotine and urine cotinine levels categorised by smoking habits of parents. Neither: Neither parents smoked in the home. Father: the father smoked while the mother did not. Mother: the mother smoked while the father did not. Both: Both parents smoked. Upper and lower whiskers represent the upper and lower confidence intervals for the respective geometric means.

excluding parents, (no smokers, one smoker, two or more smokers; Kruskal-Wallis; $\left.\chi_{\mathrm{df}=2}^{2}=30 ; \mathrm{p}<0.0001\right)$. However, there was no significant difference in hair nicotine levels according to whether there were one, two, or three or more visitors who smoked inside the house $(\mathrm{p}=0.4)$, which is probably because of the other more important sources of exposure from parents and other household members that contribute to the hair nicotine levels of children.

The variable used for ETS exposure measurement on a continuous scale was the number of cigarettes smoked by parents, adult household members, and visitors. The categories of the daily number of cigarettes smoked (1-5, 6-10, 11-15, 16-20, > $20)$ were given the values of $1,2,3,4,5$ respectively. The total number of cigarettes smoked inside the house (including cigarettes smoked by visitors) was strongly correlated with nicotine hair levels $(r=0.7)$. This correlation was slightly weaker when using only the number of cigarettes smoked by the parents at home $(r=0.68)$, which was similar to the figure obtained with the total number of cigarettes smoked by household adults (excluding the visitors).

In a grouped forward stepwise selection of the variables in the multiple regression model, the mother, father, other household adults, and visitors' number of cigarettes predicted $43 \%$ of the variability in hair nicotine levels $\left(r^{2}=0.43\right.$, $\mathrm{p}<0.0001$ ), and all variables were significant. Number of cigarettes smoked by the mother had the strongest effect on hair nicotine levels of children (coefficient estimate $(\beta)=0.5$; $\mathrm{SE}=0.05 ; \mathrm{p}<0.0001)$, followed by the father $(\beta=0.2 ; \mathrm{SE}=0.04$; $\mathrm{p}<0.0001)$, other household adult smokers $(\beta=0.1 ; \mathrm{SE}=0.03$; $\mathrm{p}<0.0001)$, and the visitors $(\beta=0.07 ; \mathrm{SE}=0.02 ; \mathrm{p}<0.0001)$. 
Table 1 Relation between reported exposure of children at home, and geometric means and $95 \%$ confidence intervals of hair nicotine and urine cotinine levels

\begin{tabular}{|c|c|c|c|c|c|c|}
\hline \multirow[b]{3}{*}{ Exposure group } & \multicolumn{6}{|c|}{ Questionnaire } \\
\hline & \multicolumn{3}{|c|}{ Nicotine } & \multicolumn{3}{|c|}{ Cotinine } \\
\hline & $\mathrm{n}$ & $\begin{array}{l}\text { Geometric } \\
\text { mean }\end{array}$ & $\begin{array}{l}95 \% \text { Confidence } \\
\text { intervals }\end{array}$ & $\mathrm{n}$ & $\begin{array}{l}\text { Geometric } \\
\text { mean }\end{array}$ & $\begin{array}{l}95 \% \text { Confidence } \\
\text { intervals }\end{array}$ \\
\hline No exposure & 101 & 0.58 & 0.49 to 0.68 & 49 & 0.44 & 0.24 to 0.80 \\
\hline Smoke outside the house & 69 & 2.63 & 2.03 to 3.40 & 44 & 4.10 & 2.33 to 7.23 \\
\hline Smoke inside the house & 127 & 5.62 & 4.60 to 6.86 & 65 & 5.30 & 3.25 to 8.63 \\
\hline
\end{tabular}

Including smoking inside or outside the house improved the model $\left(r^{2}=0.55\right)$ but made the contribution of the visitors and the father's smoking habits insignificant. Exposure from the car did not add significantly to the model, nor did exposure from outside the home.

\section{Urine cotinine}

For cotinine analysis, urine samples were collected from 158 children. The range of values for cotinine were wider than that for hair, there were 13 children with levels below the detection limit of $0.02 \mathrm{ng} / \mathrm{mg}$ creatinine, and the highest urine level recorded was $377.32 \mathrm{ng} / \mathrm{mg}$ creatinine. Urine cotinine levels varied according to the reported exposure category (KruskalWallis; $\chi_{\mathrm{df}=2}^{2}=49.5 ; \mathrm{p}<0.0001$ ). Table $\mathrm{l}$ and figure $\mathrm{l}$ display cotinine levels according to the category of exposure of the child. There is much greater overlap between the three exposure groups compared to those shown for nicotine. Univariate analysis of urine cotinine and hair nicotine among 133 children who had both biomarkers analysed show cotinine is much more widely spread (mean (95\% CI)), (11.9 (5.5 to 18.5)) than nicotine (4.9 (3.8 to 6.1)). The coefficient of variation of the mean for cotinine was 2.3 times higher than that of nicotine in this group of children. Children's cotinine levels differed according to their mothers' and fathers' smoking habits (Wilcoxon; $\chi^{2}=62.9 ; \mathrm{p}<0.0001$ and $\chi^{2}=17.1$; $\mathrm{p}<0.0001$ respectively). Figure 2 shows the mean levels for children according to whether their mothers or fathers smoked or not. The figure shows higher urine cotinine levels having high variability compared with nicotine in hair.

Urine cotinine levels were correlated with the number of smokers in the house $(r=0.59, \mathrm{p}<0.0001)$. The effect of including visitors with the total number of smokers in the house slightly strengthened the correlation to $r=0.61$.

The total number of cigarettes smoked by the parents at home was correlated with cotinine levels in urine $(r=0.67$, $p<0.0001$ ), while the total number of cigarettes smoked by the parents or the other household members (excluding the visitors) had a slightly weaker correlation $(r=0.61)$. When visitors were included, the correlation was slightly weaker $(r$ $=0.59$ ).

Only the mother's and adults' number of cigarettes and smoking inside or outside were significant in a multiple regression model $\left(r^{2}=0.31, \mathrm{p}<0.0001\right)$ (grouped forward stepwise analysis involving all variables relevant to ETS exposure of the child that were similarly used for the hair nicotine regression model).

Hair nicotine levels and urine cotinine levels were well correlated with each other $(r=0.69, \mathrm{p}<0.0001)$.

\section{DISCUSSION}

In this group of children, urine cotinine levels were more widely spread than nicotine, and were less able to differentiate groups of children according to reported exposure. The hair nicotine biomarker was better correlated to variables of reported exposure compared with urine cotinine levels, and these variables, individually and collectively, better predicted hair nicotine levels than urine cotinine.

The higher variability of urine cotinine may be attributable to true underlying variation in the exposure to ETS, or to error. Other studies have reported that cotinine levels may vary greatly among individuals with similar levels of exposure, ${ }^{23}$ possibly because of differences between individuals in metabolism and elimination. ${ }^{11}$ Moreover, the ELISA method for assaying cotinine may cross react with other nicotine metabolic products similar to cotinine. ${ }^{24}$ In our study, samples were run in duplicates for both assays, and the coefficient of variation $(\mathrm{CV})$ for cotinine was $74 \%$ while for nicotine was $10 \%$. This indicates more consistent results from the HPLC laboratory assay for hair compared with the ELISA assay for cotinine in urine.

Although children's hair nicotine and urine cotinine levels both differed according to parents' smoking, the confidence intervals around the geometric mean values show a wider variability for urine cotinine indicating a better discriminating ability of reported exposure to ETS by hair nicotine. This difference in variability does not seem to be related to the larger number of hair samples as the variability of urine cotinine and the stronger correlation of hair nicotine levels to questionnaires were consistent for the group of children with both samples (that is, similar sample size for hair nicotine and urine cotinine $n=133$ ). The larger number of hair samples collected indicates that hair samples are more readily collected than urine samples, which is an important advantage in epidemiological studies.

Although questionnaires are not "gold standards" as they are affected by many sources of measurement error, they are the most common methods for ascertaining exposure to ETS in epidemiology. This is the rationale for using questionnaires as the reference for both biomarkers in this and other studies. All determinants of ETS exposure (number of household smokers, number of cigarettes smoked by household members) were more strongly correlated with nicotine in hair than urine cotinine levels. The correlations between nicotine and cotinine and the reported number of cigarettes smoked at the child's home were similar to those reported by other investigators. ${ }^{25}$

Hair nicotine levels' closer correlation with questionnaire estimates of exposure to ETS compared with urine cotinine may be attributable to both questionnaire and hair nicotine being estimates of long term exposure, while cotinine estimates short-term exposure; and thus, is much more influenced by most recent variability of exposure. In addition, cotinine was less closely correlated with a composite variable of all sources of exposure, than with the number of cigarettes smoked at home by the parents only. This would be expected, as cotinine in urine seems to be more variable and less precise in the assessment of ETS exposures compared with nicotine in hair, and therefore may be able to predict only major sources of ETS exposure (in this case, number of cigarettes smoked by parents at home) rather than low levels of exposure (for example, visitors' smoking). 
Results from other studies support our findings. No significant difference in urine cotinine levels between children exposed to 10 cigarettes or less and those not exposed was reported by Nafstad et al, ${ }^{25}$ while they found nicotine levels in hair to be 3.4 times higher in the former group compared to the latter. The wide spread distribution of urine cotinine levels was also shown by Rylander et al ${ }^{10}$ by the overlapping cotinine levels between "exposed" and "non-exposed" children. They found among 351 children aged 4 months to 4 years that there was a wide range of cotinine concentrations in children with similar reported exposures. This variability has been also reported elsewhere. ${ }^{72627}$ Possible causes of such variability include differences in metabolism, formation and excretion of cotinine among individuals exposed to similar exposure levels. ${ }^{28}$

The multiple regression analysis for nicotine also shows smoking by the mother has the greatest influence on the model, followed by the father, household adults and visitors respectively. When the variables of the place of smoking by household members (inside or outside the house) were included in the model, the picture changed. The father's contribution to hair nicotine levels of children became nonsignificant, and so was the contribution of visitors, while smoking by other adults in the house were still significant $(p=0.045)$. Controlling for the place of smoking was more important for the father than for the mother, suggesting that fathers' smoking is more likely to be outside the house than inside it, compared with the mothers' smoking. The higher level of exposure resulting from mothers' smoking inside the house is expected as young children spend much more time around their mothers compared with their fathers. ${ }^{49}$ This also emphasises the mother's responsibility in limiting exposure of their children to ETS.

Winkelstein et $a l^{30}$ in a study looking at smoking outside versus inside the house in relation to ETS (measured by urine cotinine), found among several practices of avoiding or decreasing children's exposure to ETS that only smoking outside appeared to be effective. This effect in their study was not apparent when more than one smoker lived at home, probably because of difficulty in maintaining a smoke free home with larger number of smokers in the house. In our study, other adults' smoking habits were still significant in the model probably because of their relatively heavier smoking level.

The results in this study show children in homes with parents who smoked only outside the home had nicotine levels that were intermediate between children with no smokers living at home and those with smokers who smoked inside the house (fig 1 and table 1). Nicotine from clothes, furniture and walls, or breath of smokers may contribute to the observed levels among those children whom household members do not smoke inside the house. According to Arfi et al, ${ }^{31}$ nicotine can be detected in a room at least 12 hours after smoking has stopped. Another explanation is that the caregivers in this study were not reporting accurately about their smoking habits. Indeed, several studies have reported findings of misleading reporting by smokers of their smoking habits. ${ }^{32-34}$ Kohler et $a l^{35}$ reported that parents of ill children tend to underestimate their children's exposure to ETS. This may be related to the growing awareness of the adverse health effects of ETS exposure, especially in relation to children, whereby it has become socially unacceptable in some people's eyes to expose children to ETS. ${ }^{36}$

Smoking in the car was not significant in the model; which has been also reported by Henderson et al. ${ }^{9}$ In our study most of the parents reported that they opened the car window if they smoked in the car, which could explain the lack of effect as a result of good ventilation leading to insignificant levels of exposure.

In conclusion, when compared with questionnaire reports of ETS exposure, the hair nicotine method used in our study of assessment of ETS exposure among young children was more

\section{Key points}

- The hair nicotine method is an accurate measure of ETS exposure among children.

- It is more precise than currently used urine cotinine and more easily collected.

- It has numerous public health implications in monitoring, and cessation of tobacco smoke exposure.

precise than the ELISA urine cotinine method for the same individuals. Using several indicators of ETS exposure for the purpose of comparison yielded similar results in support of the superiority of hair nicotine as a biomarker of ETS exposure in the largest study to assess this biomarker yet. The ability of long term exposure measurement seems to overcome the wide variability introduced by short-term irregular ETS exposure from the environment.

A "smoke-free house" in this study minimised but did not completely protect the children against exposure to ETS, and the best approach to prevent exposure of children is cessation of smoking by household members, especially mothers.

\section{ACKNOWLEDGEMENTS}

Funding: we would like to acknowledge the Hawke's Bay Medical Research Foundation, Napier, New Zealand for providing the funding for this study.

Conflicts of interest: none.

\section{Authors' affiliations}

W K Al-Delaimy, A Woodward, Department of Public Health,

Wellington School of Medicine, Wellington, New Zealand

J Crane, Department of Medicine, Wellington School of Medicine

\section{REFERENCES}

1 EPA. Respiratory health effects of passive smoking: lung cancer and other disorders. Bethesda, MA: Environmental Protection Agency, 1993.

2 Benowitz NL. Cotinine as a biomarker of environmental tobacco smoke exposure. Epidemiol Rev 1996;18:188-204.

3 Forastiere F, Agabiti N, Dell'Orco V, et al. Questionnaire data as predictors of urinary cotinine levels among nonsmoking adolescents. Arch Environ Health 1993:48:230-4.

4 Bono R, Russo R, Arossa W, et al. Involuntary exposure to tobacco smoke in adolescents: urinary cotinine and environmental factors. Arch Environmenl Health 1996;51:127-31.

5 Willers S, Skarping G, Dalene $M$, et al. Urinary cotinine in children and adults during and after semi-experimental exposure to environmental tobacco smoke. Arch Environ Health 1995;50: 130-8.

6 Margolis PA, Keyes LL, Greenberg RA, et al. Urinary cotinine and parent history (questionnaire) as indicators of passive smoking and predictors of lower respiratory illness in infants. Pediatr Pulmonol 1997:23:417-23.

7 Peterson EL, Johnson CC, Ownby DR. Use of urinary cotinine and questionnaires in the evaluation of infant exposure to tobacco smoke in epidemiologic studies. J Clin Epidemiol 1997;50:917-23.

8 Greenberg RA, Haley NJ, Etzel RA, et al. Measuring the exposure of infants to tobacco smoke. Nicotine and cotinine in urine and saliva. $N$ Engl J Med 1984;310:1075-8.

9 Henderson F, Reid H, Morris R, et al. Home air nicotine levels and urinary cotinine excretion in preschool children. Am Rev Respir Dis 1989; 140:197-201.

10 Rylander E, Pershagen G, Eriksson $M$, et al. Parental smoking, urinary cotinine, and wheezing bronchitis in children. Epidemiology 1995:6:289-93

11 Idle JR. Titrating exposure to tobacco smoke using cotinine-a minefield of misunderstandings. J Clin Epidemiol 1990;43:313-17.

12 Sepkovic DW, Haley NJ. Biomedical applications of cotinine quantitation in smoking related research. Am J Public Health 1995;75:663-5

13 Dvorchick BH, Vesell ES. Pharmacokinetics interpretation of data gathered during therapeutic drug monitoring. Clin $\mathrm{Chem}$ 1976;22:868-78.

14 Jarvis MJ, Russell MAH, Feyerabend $C$, et al. Passive exposure to tobacco smoke: saliva cotinine concentration in a representative population sample of non-smoking schoolchildren. BM 1985;291:927-9.

15 Coultas DB, Howard CA, Peake GT, et al. Salivary cotinine levels and involuntry tobacco smoke exposure in children and adults in New Mexico. Am Rev Respir Dis 1987;136:305-9. 
16 Ronchetti R, Bonci E, Martinez FD. Passive smoking in childhood-tobacco smoke. Lung 1990;168:313-19.

17 Uematsu T, Mizuno A, Nagashima S, et al. The axial distribution of nicotine content along hair shaft as an indicator of changes in smoking behaviour: evaluation in a smoking-cessation programme with or without the aid of nicotine chewing gum. Br J Clin Pharmacol 1995;39. 665-9

18 Jurado C, Kintz P, Menendez $M$, et al. Influence of the cosmetic treatment of hair on drug testing. Int J Legal Med 1997;1 10:159-63.

19 Mahoney GN, Al-Delaimy WK. The measurement of nicotine in hair by reversed-phase high performance liquid chromatography with electrochemical detection. J Chromatog Biomed B Appl 2001;753:179-87

20 Ford $\mathbf{R}$, Tappin D, Schluter $P$, et al. Smoking during pregnancy: how reliable are maternal self-reports in New Zealand? J Epidemiol Community Health 1997;51:246-51.

21 Tappin D, Ford R, Schluter P. Smoking during pregnancy measured by population cotinine testing. NZ Med J 1997;1 10:311-14.

22 Galanti LM, Dell'Omo J, Vanbeckbergen D, et al. Particle counting immunoassay for urinary cotinine. Comparison with chromatography, enzyme-linked immunoassay and fluorescence polarization immunoassay. Clin Chem Lab Med 1999;37:729-34

23 Cholerton S, Ayesh R, Idle J, et al. The pre-eminence of nicotine $\mathrm{N}$-oxidation and its diminution after carbimazole adminstration. $\mathrm{Br} J \mathrm{Clin}$ Pharmacol 1988;26:652-3.

24 Schepers G, Walk R. Cotinine determination by immunoassays may be influenced by other nicotine metabolites. Arch Toxicol 1988;62:395-7.

25 Nafstad P, Botten G, Hagen JA, et al. Comparison of three methods for estimating environmental tobacco smoke exposure among children aged between 12 and 36 months. Int J Epidemiol 1995;24:88-94.

26 Etzel R. A review of the use of saliva cotinine as a marker of tobacco smoke exposure. Prev Med 1990;19:190-7.
27 Pirkle JL, Flegal KM, Bernert JT, et al. Exposure of the US population to environmental tobacco smoke: the Third National Health and Nutrition Examination Survey, 1988 to 1991. JAMA 1996;275:1233-40.

28 Fernandez-Salguero P, Hoffman SMG, Cholerton S, et al. A genetic polymorphism in coumarin 7-hydroxilation: sequence of the human CYP2A genes and identification of variant CYP2A6 alleles. Am J Human Genet 1995; 57:651-60.

29 Knight JM, Eliopoulos C, Klein J, et al. Passive smoking in children. Racial differences in systemic exposure to cotinine by hair and urine analysis. Chest 1996;109:446-50.

30 Winkelstein ML, Tarzian A, Wood RA. Parental smoking behavior and passive smoke exposure in children with asthma. Ann Allergy Asthma Immunol 1997;78:419-23.

31 Arfi C, Kaloustian J, Pauli A, et al. Nicotine and indoor air pollution. In: Bieva C, Courtois Y, Govaerts M, eds. Present and future of indoor air Quality. Brusseles: Excerpta Medica, International Congress Series, Elseiver Science, 1989.

32 Sillett RW, Wilson MB, Malcolm RE, et al. Deception among smokers. BM 1978;2:1185-6.

33 Jarvis MJ, Tunstall-Pedoe H, Feyerabend C, et al. Comparison of tests used to distinguish smokers from non-smokers. Am J Public Health 1987;77: 1435-8.

34 Heller WD, Scherer G, Sennewald E, et al. Misclassification of smoking in a follow-up population study in southern Germany. J Clin Epidemiol 1998;51:211-18.

35 Kohler E, Sollich V, Schuster R, et al. Passive smoke exposure in infants and children with respiratory tract diseases. Hum Exp Toxicol 1999;18:212-217.

36 AL-Delaimy W, Lou D, Woodward A, et al. Smoking hygience: a study of attitudes to passive smoking. NZ Med J 1999;113:33-6.

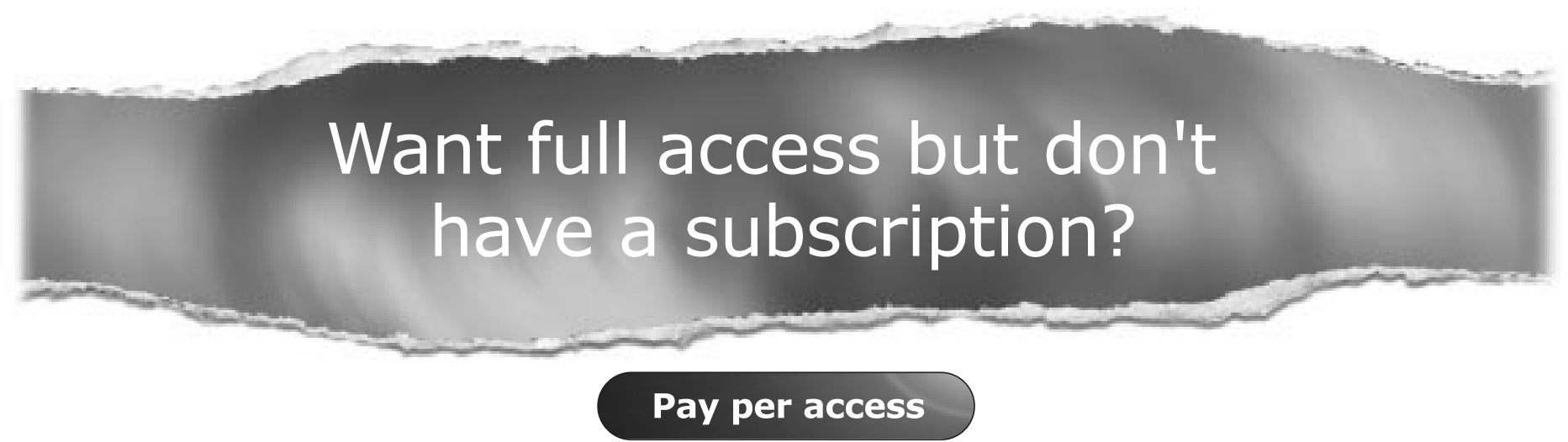

For just US $\$ 25$ you can have instant access to the whole website for 30 days. During this time you will be able to access the full text for all issues (including supplements) available. You will also be able to download and print any relevant pdf files for personal use, and take advantage of all the special features Journal of Epidemiology and Community Health online has to offer.

\section{www.jech.com}

